\title{
The Influence of Inequality, Responsibility and Justifiability on Reports of Group-Based Guilt for Ingroup Privilege
}

\author{
Robyn K. Mallett \\ The University of Virginia
}

\section{Janet K. Swim}

The Pennsylvania State University

\begin{abstract}
Although members of several social groups report feeling guilt because of their group's actions, average reports of group-based guilt tend to be quite low. We investigate three antecedents of group-based guilt derived from research on social justice and interpersonal emotion. We find that Whites, men and women perceive inequality, responsibility and justifiability of group differences to the same extent. Moreover, each factor is a key antecedent of guilt for Whites, men and women. We also find an interaction between justifiability and responsibility such that reports of group-based guilt increase as perceptions of ingroup responsibility increase and justifications for group differences decrease. Given the beneficial consequences of group-based guilt for intergroup relations, it is important to understand what factors lead to group-based guilt.
\end{abstract}

KEYWORDS group-based guilt, inequality, ingroup responsibility, justifiability, men, Whites, women

Although some individuals report feeling guilt because of their group's actions, average reports of group-based guilt are often quite low (Swim \& Miller, 1999). Yet, when experienced, group-based guilt can motivate beneficial social action including apology for past acts committed by ingroup members (Doosje, Branscombe, Spears, \& Manstead, 2004) and majority group member participation in collective action on behalf of disadvantaged social groups (Mallett, Huntsinger, Sinclair, \& Swim, 2006). Research has yet to clarify exactly why reports of group-based guilt tend to be low, but it has established that at least two factors are critical antecedents of guilt at the group level (Branscombe, Doosje, \& McGarty, 2003). First, there must be an apparent inequality between groups which could be the

\footnotetext{
$\overline{\text { Author's note }}$

Address correspondence to Robyn Mallett, Department of Psychology, University of Virginia, 102 Gilmer Hall, P.O. Box 400400, Charlottesville, VA 22904-4400, USA

[email: mallett@virginia.edu]
} 
result of long-term disadvantage or of a onetime critical incident. Second, the ingroup must be seen as responsible for the inequality. Some research also indicates that justifications for inequality may reduce feelings of group-based guilt (Miron, Branscombe, \& Schmitt, 2006). Past research has routinely assessed potential antecedents of group-based guilt in isolation, thereby failing to provide a complete picture of the relative strength of key antecedents. Moreover, the majority of past research has examined feelings of guilt in only one group at a time, leaving unanswered the question of whether relations among the antecedents and groupbased guilt differ between social groups. We synthesize the existing research by simultaneously examining three antecedents of group-based guilt in Whites, men and women.

\section{Antecedents of group-based guilt}

\section{Perceived inequality}

Research on social justice and interpersonal emotions reveals that personal feelings of guilt can result when people perceive that they are over-benefited or have harmed another person (Austin \& Walster, 1974; Baumeister, Stillwell, \& Heatherton, 1994; Sprecher, 1986; Walster \& Walster, 1975; Walster et al., 1978). Similarly, group-based feelings of guilt can result from perceived or actual intergroup inequality (Branscombe et al., 2003), of which there is ample evidence (e.g. Anderson, 1989; Russo, 1995; Swim, Aikin, Hall, \& Hunter, 1995; Turner \& Kramer, 1995). Data supporting the connection between perceived inequality and group-based guilt exists for a variety of groups, including Whites' reports of group-based guilt for White privilege and Black disadvantage (Swim \& Miller, 1999), Germans' reports of guilt for enacting genocide against the Jews (Rensmann, 2004), and men's reports of guilt for privileges they have over women (Schmidt, Branscombe, \& Brehm, 2004).

The present research tests three hypotheses related to this well-established antecedent. First, we predict that the more a group difference is perceived to be important and advantageous to the ingroup, the more likely individuals will be to report group-based guilt. We assume that people will be able to recognize that a group difference exists when it is brought to their attention. But even when faced with examples of group differences that favor the ingroup, some individuals may not report group-based guilt. To uncover when perceived inequality is related to guilt, we assess the magnitude of perceived inequality by measuring the perception that a group difference is important and provides an advantage to the ingroup (Lerner, 1981). Although women may acknowledge that they are more likely to receive assistance with car trouble than men, if women do not perceive that vehicular assistance is important or that it gives women an advantage over men, then they may not feel particularly guilty about that group difference. In contrast, if Whites perceive their greater likelihood of having an economic safety net than Blacks is important and advantageous, they might report feeling group-based guilt.

Second, we hypothesize that perceived inequality will uniquely predict reports of groupbased guilt above and beyond perceptions of responsibility and justifiability. Third, we test whether perceived inequality is a key antecedent of group-based guilt for members of three different social groups. To our knowledge, ours is the first research to examine the antecedents of group-based guilt among members of a disadvantaged group (i.e. women). We expect that perceptions of inequality will influence reports of guilt to the same extent across groups. It is possible, however, that the strength of the association between perceived inequality and guilt will vary by group membership-particularly if perceived inequality resulting from ingroup privilege is construed in the larger social context. For example, to the extent that women's advantages are tied to their dependency on men (e.g. being helped by men) or to traditional gender roles that maintain the existing social order (e.g. being awarded child custody), women may perceive that a group difference produces little inequality because it comes at a cost to women. Or it could lead to the assessment of inequality having less of an impact on women's 
group-based guilt. Therefore, the impact of perceived inequality on feelings of group-based guilt might differ depending on the group under consideration.

\section{Perceived responsibility}

Consistent with past research we predict that the more the ingroup is perceived to be responsible for their privileges, the more likely people will be to report group-based guilt. Personal guilt results when people accept responsibility for an action or inaction that caused another to suffer (Estrada-Hollenbeck \& Heatherton, 1998; Hoffman, 1994), and group-based guilt results when people accept ingroup responsibility for harm inflicted on the outgroup (Branscombe et al., 2003; Branscombe, Slugoksi, \& Kappen, 2004; Iyer, Leach, \& Pederson, 2004). We conceive of responsibility as being composed of both accountability for creating a group difference, which implies that responsibility is in the past, and accountability for maintaining a group difference, which implies that responsibility continues to the present day. Feelings of group, but not personal responsibility, are positively related to reports of group-based guilt for both minimal groups (Doosje, Branscombe, Spears, \& Manstead, 1998) and a variety of existing social groups (Branscombe, Doosje \& McGarty, 2002).

We test two hypotheses related to perceptions of ingroup responsibility. First, we test whether ingroup responsibility predicts feelings of group-based guilt above and beyond perceived inequality and justifiability. Existing research that measures or manipulates group-based guilt generally confounds inequality and responsibility. For example, items from Branscombe and colleagues' (2004) collective guilt acceptance scale invokes group responsibility and resulting inequality in the same statement, 'I feel regret for my group's harmful past actions toward other groups'. Determining the extent to which guilt results from ingroup responsibility versus perceived inequality would further clarify the construct of group-based guilt. Discovering the unique predictive ability of responsibility is an especially worthy goal considering that not all research has found that accepting responsibility is necessary to feel guilt. For instance, Hoffman (1994) describes two guiltproducing situations, the innocent bystander and the virtual transgressor, where the individual is not necessarily responsible for a transgression but feels guilt anyway. Although Hoffman did not consider group-based guilt, his analysis indicates that responsibility may not play as essential a role as other research indicates.

Second, we test whether perceived ingroup responsibility is perceived to the same extent across groups and has the same relation with guilt for Whites, men and women. As with perceived inequality, we expect that perceptions of responsibility will influence reports of guilt to the same extent across groups, but it is also possible that the strength of the association between responsibility and guilt will vary by group membership. For example, because gender differences, more so than racial differences, may be attributed to biological imperatives and the socially accepted complementarity of group roles, assessments of responsibility may differ or responsibility may play less of a role in predicting gender-based guilt than race-based guilt.

\section{Perceived justifiability}

Most discussions of group-based guilt do not include assessments of the justifiability of group differences as a required antecedent, but research on social justice suggests that justifications for group differences could influence the level of guilt experienced (Lerner, 1981; Montada \& Schneider, 1989; Tyler, Boeckmann, Smith, \& Huo, 1997). At the individual level, guilt results if an act violates personal standards for justice or morality (Harder \& Greenwald, 1999; Monteith, Devine, \& Zuwerink, 1993; Roseman, 1984), and at the group level, perceived justifiability of group differences reduces the overall experience of guilt (Branscombe \& Miron, 2004). Based upon this research, we anticipate that perceived justifiability will be an important antecedent of group-based guilt.

We test two hypotheses relevant to perceptions of justifiability. First, since past research has rarely considered perceptions of justifiability, we test whether justifiability uniquely predicts feelings 
of group-based guilt when perceived inequality and responsibility are also assessed. Second, we test whether perceived justifiability is perceived to the same extent and has the same relation to guilt across three different social groups. Similar to perceived responsibility, beliefs about innate gender differences and gender roles may be used to legitimize gender group differences (Glick \& Fiske, 1997). Thus, justifications generated for gender may be perceived as more credible than justifications generated for race and may therefore have more influence in diminishing feelings of gender-based guilt.

\section{Interactions between perceived inequality, responsibility and justifiability}

The above analysis indicates that perceptions of inequality and ingroup responsibility will increase feelings of group-based guilt and that perceptions of justifiability will decrease feelings of group-based guilt. It is possible that the three antecedents are additive, such that more inequality and responsibility and less justifiability produce the most guilt. Alternatively, if any of the three factors are necessary conditions to experience guilt, then they should interact with the others over and above their additive effects. Below we outline possible patterns of interactions that might emerge.

Perceived inequality may be a necessary antecedent of group-based guilt, as it might first be necessary to decide that a group difference exists before assessing responsibility or justifiability. Even if a group difference is acknowledged, responsibility and justifiability may not strongly influence group-based guilt unless the difference is perceived to be important and advantageous. For instance, if women perceive that being less likely than men to pay for a romantic evening is not important or advantageous, whether or not women perceive that they are responsible for the difference or that it is justifiable, they may not feel particularly guilty. Thus, perceived inequality and perceptions of responsibility and justifiability may interact such that as perceptions of inequality increase, perceptions of responsibility and justifiability are stronger predictors of group-based guilt.

When group differences are perceived to be important and advantageous, perceived justifications may diminish the impact of responsibility on feelings of group-based guilt. For example, if women perceive that women receiving custody of children after a divorce is justified because of innate gender differences in the ability to nurture and parent, then women's perceptions of responsibility for receiving custody may have less influence on guilt than if receiving custody was not perceived to be justified. Thus, we may find a three-way interaction between inequality, justifiability and responsibility such that group-based guilt only occurs when group differences are perceived to produce inequality, are unjustified and the ingroup is perceived to be responsible.

\section{Goals of the present research}

With the goal of better understanding what factors lead to feelings of group-based guilt, we asked participants to read descriptions of several group differences that favored their ingroup. For example, women read examples of group differences that favored women and men read examples of group differences that favored men. Participants rated the extent to which they perceived that each difference was important and advantageous (i.e. extent of inequality), that their group was responsible for creating and maintaining each difference (i.e. extent of responsibility), that each difference was justifiable, and that they felt group-based guilt for each difference. First, we tested whether the three groups perceived the antecedents and group-based guilt in the same manner. Second, we tested whether the relations between the antecedents and group-based guilt differed by group membership. Third, we tested whether each factor was a unique antecedent of groupbased guilt. Specifically, we predicted that the more inequality and responsibility participants perceived and the less justifiability they perceived, the more they would report group-based guilt. Fourth, although we expected to find mainly 
additive effects, we also explored whether there were interactions between the antecedents and group-based guilt.

\section{Method}

\section{Participants}

We tested our hypotheses in three samples. Participants from all three samples were enrolled in undergraduate psychology classes at the Pennsylvania State University. Participants for the White sample included 38 males and 96 females, ranging in age from 18 to $46(M=18.9, S D=3.0)$. All participants reported their race/ethnicity as White. Participants for the sample of men included 115 men, ranging in age from 18 to $23(M=19.4, S D=1.4) ; 5 \%$ of the sample were Asian, 2.6\% were Black, 2.6\% were Hispanic, $88.7 \%$ were White and less than $1 \%$ chose the label of something else. Participants for the sample of women included 109 women, ranging in age from 18 to $37(M=19.7, S D=3.8) ; 1 \%$ of the sample were American Indian, $7.3 \%$ were Asian, $10.9 \%$ were Black, $2.7 \%$ were Hispanic, $76.4 \%$ were White and $1.8 \%$ designated their background as something else. All participants completed materials for this study on the Internet.

\section{Materials and procedure}

Participants first provided demographic information and then evaluated a series of group differences that pertained to their ingroup only. For example, Whites only read about group differences that favored Whites. Salient group membership is critical to producing feelings of group-based guilt (Branscombe, Doosje \& McGarty, 2002). We ensured that race was salient for the sample of Whites and gender was salient for the samples of women and men by having all participants indicate their race or gender at the beginning of the survey and by highlighting the social group of interest in each item. For example, White participants saw the phrase 'Whites, as a group' 114 times while completing the study measures.

Gender differences were selected from Branscombe's (1998) list of gender privileges and we generated some of our own examples to cover aspects of gender privilege that were not represented. We provided 20 group differences for women, and 21 group differences for men. Gender differences included the following categories:

- Gender roles (e.g. women are more likely than men to have people stop and offer assistance with car trouble/women are more likely than men to have to do housework).

- Treatment by authorities (e.g. women are more likely than men to pay less for auto insurance/ men are more likely than women to get fair estimates for auto repairs).

- Financial (women are less likely than men to pay for a date/men are more likely than women to be given fair career opportunities).

- Freedom (e.g. women are less likely than men to appear weak when they cry/men are less likely than women to be afraid of being sexually harassed or raped).

- Special social treatment (e.g. women are more likely than men to receive flowers/men are more likely than women to receive special help from professors on difficult material).

- Help from others (e.g. women are less likely than men to be asked to lift heavy objects/men are less likely than women to be expected to take time off of work to care for others such as children or elderly members of one's family).

Some examples of group differences for Whites ( $n=19)$ were selected from McIntosh's (1998) list of White privileges. Again, we generated additional items to cover unrepresented areas. Racial differences included the following categories:

- Financial (e.g. Whites are more likely than Blacks to have an economic 'safety net' to fall back on in times of financial trouble).

- Historical distortion (Whites are more likely than Blacks to have historical struggles represented in history books).

- Social treatment (Whites are more likely than Blacks to be able to be able to complain about colleague or coworker behaviors without it 
being assumed that the matter is a racial issue).

- Political (e.g. Blacks are more likely than Whites to have skin color taken into account when police decide whether to perform a routine traffic stop).

After reading each group-specific example, participants first evaluated inequality by indicating how important the difference was (e.g. it is an important group difference within society, regardless of whether it favors men or women) and how advantageous the difference was for their group (e.g. it is actually an advantage that men have relative to women). Participants then evaluated how justifiable the difference was (i.e. it is justifiable or fair). Next, participants evaluated the extent to which their ingroup was responsible for creating (e.g. Whites are the source of the advantage) and maintaining (e.g. Whites maintain the advantage) the group difference. Finally each group-specific difference was evaluated for how the difference made them feel (e.g. guilty that men have this advantage relative to women). All responses were made on a 1 not at all to 4 very much scale. We standardized all variables before conducting the analyses reported below.

\section{Results}

The data for the present research were nested within participants; therefore the ratings of each group difference were not independent. To account for the interdependence, we used Hierarchical Linear Modeling to conduct multilevel modeling (Raudenbush, Bryk, Cheong, \& Congdon, 2004) and test the extent to which our proposed antecedents predict group-based guilt. An additional advantage of multilevel modeling is that we can test for cross-level effects, or interactions, between groups and ratings of the group differences. In the present study, this means that we can test whether there are between-group differences in the extent to which the proposed antecedents predict group-based guilt. For instance, we can test whether the association between perceived responsibility and group-based guilt is equally strong for Whites, men and women. Additionally, crosslevel analyses control for main effects of group membership on the ratings. Because we standardized all items, the $\mathrm{G}$ coefficient in the analyses reported below can be interpreted similarly to a standardized beta weight in regression.

\section{Preliminary analyses}

Group differences in evaluations of the antecedents and reports of guilt We first constructed three dummy-coded contrasts to test whether evaluations of the antecedents and reports of guilt differed by group. One contrast compared Whites (2) versus men $(-1)$ and women $(-1)$. The second compared men (2) versus Whites $(-1)$ and women $(-1)$. The third compared women (2) versus Whites $(-1)$ and men $(-1)$. We then tested whether each contrast predicted each antecedent by entering the contrast as a level 2 predictor of each antecedent's intercept. None of the contrasts significantly predicted the intercept for inequality $(G=-.002, S E=.02$, $p=n s, G=-.004, S E=.02, p=n s, G=-.001$, $S E=.02$; Whites, men, women, respectively), responsibility $(G=-.004, S E=.02, p=n s, G=-.007$, $S E=.03, p=n s, G=-.002, S E=.02, p=n s$; Whites, men, women, respectively), or justifiability $(G=-.001, S E=.02, p=n s, G=-.03, S E=.02$, $p=n s, G=.003, S E=.02, p=n s$; Whites, men, women, respectively). Additionally, none of the contrasts significantly predicted the intercept for guilt $(G=-.009, S E=.03, p=n s, \mathrm{G}=.02$, $S E=.03, p=n s, G=-.009, S E=.03, p=n s$; Whites, men, women, respectively). Therefore, Whites, men and women all perceived inequality, responsibility and justifiability, and reported group-based guilt, to the same extent. ${ }^{1}$

Correlations between the antecedents We first investigated the appropriateness of combining the advantageousness and importance items to create a measure of inequality. The two evaluations are correlated, yet distinct at $G=.25$, $S E=.01, p<.001$. When examined separately, we find the same pattern of results reported below for advantageousness and importance as for the composite measure of inequality. 
We then investigated the appropriateness of combining the evaluations of accountability for creating and maintaining a group difference to create a measure of responsibility. The association between ratings of accountability for creating and maintaining group differences was strong $(G=.73, S E=.01, p<.001)$, suggesting that the distinction was not meaningful in the present study. Therefore we averaged the two measures together. When examined separately, we find the same pattern of results reported below for both types of responsibility as for the composite measure.

We next assessed the correlations between the antecedents by entering one antecedent as the dependent variable and a second antecedent predicting that variable at level 1 . In order to control for group membership, in separate analyses, we also entered each contrast as a level 2 predictor of the dependent variable. We found that the associations were identical when controlling for each contrast. Perceived inequality was correlated with perceived responsibility $(G=.24, S E=.01$, $p<.001)$ and perceived justifiability $(G=.25$, $S E=.01, p<.001)$. Perceived justifiability was correlated with perceived responsibility $(G=.06$, $S E=.01, p<.001)$.

\section{Main effects}

We expected that each antecedent would be uniquely associated with group-based guilt, but that the strength of the associations might differ by group. We tested these two questions by entering inequality, responsibility and justifiability as level 1 predictors of group-based guilt. In three separate analyses, we entered the contrasts from above as a level 2 predictor of group-based guilt and of the slope for each predictor. For instance, we included the contrast between Whites versus men and women as a predictor of group-based guilt and the same contrast as a predictor of the slope measuring the association between perceived inequality and group-based guilt. Consistent with the lack of group differences reported above for groupbased guilt, none of the contrasts predicted the intercept $(G=-.008, S E=.02, p=n s, G=.02$, $S E=.02, p=n s, G=-.007, S E=.02, p=n s$; Whites, men, women, respectively). However we did find that the strength of associations between guilt and both inequality and responsibility differed on one or more contrasts. Specifically, the association between inequality and guilt differed for the contrast comparing women to Whites and men. Therefore we analyzed the association between inequality and guilt separately for each group and found that inequality was significantly related to reports of group-based guilt for all three groups, but the association between inequality and guilt was stronger for Whites $(G=.18, S E=.02, p<.001)$ and men $(G=.18$, $S E=.02, p<.001)$, compared to women $(G=.06$, $S E=.02, p<.01)$. The association between responsibility and guilt differed for all three contrasts $(G=.05, S E=.01, p<.001, G=.03$, $S E=.01, p<.001, G=-.08, S E=.01, p<.00$, Whites, men, women respectively). When we analyzed the association between responsibility and guilt separately for each group we found that, as with perceived inequality, the association between responsibility and guilt was significant for all three groups, but the association was stronger for Whites $(G=.33$, $S E=.02, p<.001)$ and men $(G=.30, S E=.02$, $p<.001)$, compared to women $(G=.09, S E=.02$, $p<.001)$. There were no cross level effects for the association between justifiability and guilt, but justifiability was significantly associated with guilt and the association did not differ by contrast $(G=-.12, S E=.01, p<.001)$.

\section{Interactions}

Finally, we tested for two- and three-way interactions between inequality, responsibility and justifiability. At level 1, we entered inequality, responsibility and justifiability, as well as the two-way interactions between inequality and responsibility, inequality and justifiability, and responsibility and justifiability, and the threeway interaction. In three separate analyses we entered each contrast as a level 2 predictor of group-based guilt and of the slope for the main effects, two-way and three-way interactions. The contrasts did not predict the slope for the three-way interaction. An analysis without the contrast predicting the slope showed that 
the three-way interaction was not significant $(G=.007, S E=.007, p=n s)$.

We then removed the three-way interaction from the equation and tested whether the contrasts predicted the two-way interactions. At level 1 , we entered inequality, responsibility and justifiability, the two-way interactions between inequality and responsibility, inequality and justifiability, and responsibility and justifiability. At level 2, in three separate analyses we entered each contrast as a predictor of groupbased guilt and of the slope for the main effects and the two-way interactions. None of the contrasts predicted the slopes of the two-way interactions between inequality and responsibility or between inequality and justifiability. When the contrast was not included as a predictor of the interactions, the interactions remained nonsignificant, indicating that there was no two-way interaction for inequality, and this was true for all three groups.

The interaction between justifiability and responsibility was significant. Further, the contrast predicting the slope of the interaction was significant for Whites versus men and women $(G=-.02, S E=.01, p=.004)$ and men versus Whites and women $(G=.02, S E=.01, p=.002)$, but not for women versus Whites and men $(G=-.005, S E=.006, p=n s)$. Because two of the three contrasts were significant, we analyzed the interaction separately for each group by including justifiability, responsibility and their interaction as level 1 predictors of group-based guilt. The interaction was significant for Whites $(G=-.08, S E=.01, p<.001)^{2}$ and women $(G=$ $-.05, S E=.02, p=.02)$, but not for men $(G=.002$, $S E=.02, p=n s)$.

We expected that responsibility would be a stronger predictor of guilt when justifiability was low and when inequality was high. The three-way interaction did not support this effect; however by extension we would predict the same pattern for a two-way interaction between justifiability and responsibility. Consistent with this prediction, an examination of the means indicates that the most guilt was experienced when responsibility was high and justifiability was low. Figures $1 \mathrm{a}$ and $1 \mathrm{~b}$ show the pattern of the interaction for Whites and women. We also examined the simple effects for responsibility predicting guilt at low, medium and high levels of justifiability and for justifiability predicting guilt at low, medium and high levels of responsibility. For Whites, responsibility predicted guilt at all levels of justifiability, but was slightly stronger when justifiability was low $(\hat{\omega}=.46(.05)$, $t=20.42, p<.001)$ than when justifiability was medium $(\omega \dot{\omega}=.38(.02), t=21.05, p<.001)$ or high $(\dot{\omega}=.29(.02), t=13.66, p<.001)$. Further, justifiability predicted group-based guilt when responsibility was medium $\left(\omega^{\prime}=.11(.02)\right.$, $t=7.52, p<.001)$ and high $\left(\omega^{\prime}=.19(.02)\right.$,

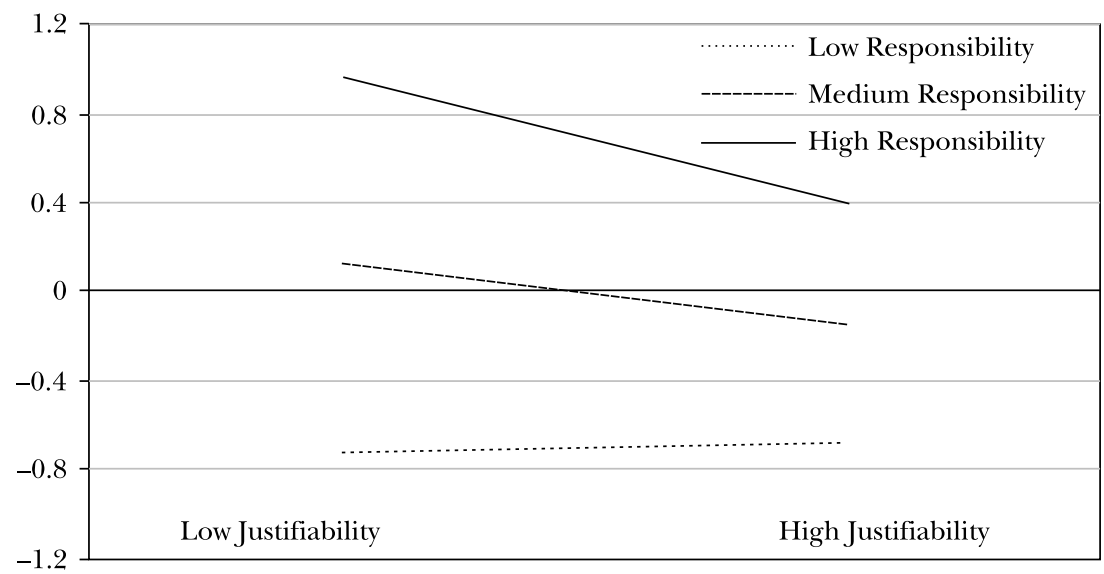

Figure 1a. The two-way interaction between justifiability and responsibility for reports of White guilt. 


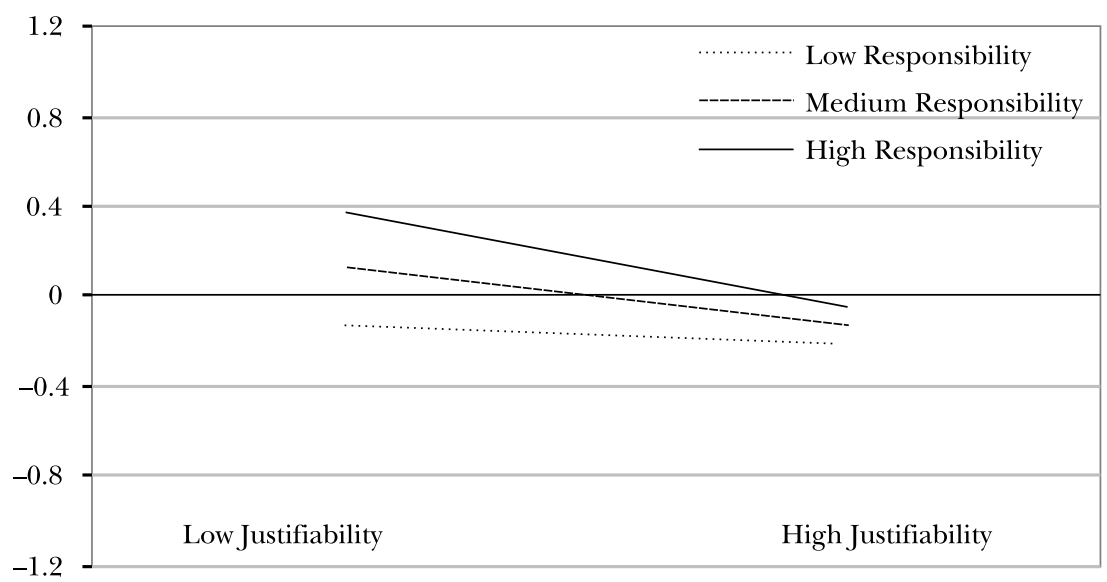

Figure $1 b$. The two-way interaction between justifiability and responsibility for reports of women's genderbased guilt.

$t=10.31, p<.001)$, but not when responsibility was low $(\omega=.03(.02), t=1.43, p=n s)$. For women, responsibility predicted guilt when justifiability was low $(\dot{\omega}=.17(.04), t=4.46$, $p<.001)$ and medium ( $\dot{\omega}=.08(.02), t=4.19$, $p<.001)$, but not when justifiability was high ( $\left.\omega^{\prime}=-.01(.04), t=-.17, p=n s\right)$. Replicating the results for Whites, justifiability predicted group-based guilt when responsibility was medium $(\dot{\omega}=-.12(.06), t=-2.04, p=.04)$ and high ( $\left.\omega^{\prime}=-.22(.07), t=-3.12, p=.002\right)$, but not when responsibility was low $\left(\omega^{\prime}=-.04(.07)\right.$, $t=-.53, p=n s)$.

\section{Discussion}

The present research makes several important contributions to the literature on group-based guilt. First, we find that Whites, men and women perceive the same extent of inequality, responsibility, justifiability and group-based guilt. This is interesting given the fact that women are a low status, disadvantaged group, and might be expected to view their privileges as compensation for their low social status, thereby attenuating the influence of the antecedents on group-based guilt or reporting less guilt.

Second, we find that inequality, responsibility and justifiability are each unique and significant predictors of group-based guilt for Whites, men and women. When considering differences that favor the ingroup, people need to go beyond merely recognizing that a difference exists; they need to acknowledge the added advantage and importance that the difference provides for the ingroup in order to feel group-based guilt. We also find that for all three groups, perceptions of ingroup responsibility were positively related to reports of group-based guilt and that the justifications for group differences were negatively related to reports of group-based guilt. This is the first research to establish the unique predictive ability of justifiability in the context of inequality and responsibility. Thus consistently across groups, we find reliable additive effects of perceived inequality, responsibility and justifiability of group differences for reports of group-based guilt.

Third, we find that although all three antecedents were significantly associated with reports of group-based guilt, the strength of some associations differed by group. Perceived inequality was a stronger predictor of groupbased guilt for Whites and men than for women. Although possibly due to the types of privileges that participants considered, the strength of the association between inequality and guilt might have been weaker for women because women perceived their privileges within the context of larger social inequality. Perceptions of ingroup 
responsibility were also a stronger predictor of guilt for Whites than for men and women. The association between responsibility and genderbased guilt, compared to race-based guilt, might have been weakened if gender differences were attributed to biology and complementarity more so than racial differences.

Fourth, we find an interaction between justifiability and responsibility for women and Whites but not for men, indicating that for men, responsibility and justifiability independently predict gender-based guilt. But when Whites considered race-based guilt and when women considered gender-based guilt, responsibility played a greater role when group differences were not very justifiable. Because the influence of responsibility on group-based guilt was tempered by justifications for ingroup privilege, a lack of justifications for privilege is also necessary for feelings of group-based guilt in Whites and women. Interestingly, the effect of perceived inequality was not moderated by perceived ingroup responsibility or justifications for ingroup privilege. Therefore, for at least some people, feelings of group-based guilt do not require perceived ingroup responsibility or a lack of justifications for ingroup privilege. This finding is in line with Hoffman's (1994) work on feelings of guilt at the individual level—especially situations where people are innocent bystanders or a virtual transgressor. In both cases, the individual is not necessarily responsible for a transgression but feels guilt anyway.

It is difficult to know precisely why the interaction between justifiability and responsibility only occurred for Whites and women. Perhaps men occupy a unique position as members of an advantaged gender group. Future research should sample a wider variety of groups to determine exactly when the antecedents are additive versus interactive as different types of advantaged groups, including heterosexuals or able-bodied individuals, may also differ in the experience of guilt. We anticipate that perceived inequality, responsibility and justifiability will still predict group-based guilt, but that evaluations of the antecedents might vary. Finally, future research could continue to test our model by examining how other disadvantaged groups, including
Blacks, non-heterosexuals and the disabled, assess their privileges. Other disadvantaged groups' privileges may be qualitatively different from those experienced by women, given that women's privileges are largely based upon gender role norms.

It would also be interesting to consider additional antecedents of guilt that operate at the interpersonal level. We chose to examine antecedents of guilt that operate regardless of the relationship between social groups, but Baumeister and colleagues (1994) describe antecedents of guilt that appear in close, communal relationships where two individuals are concerned about each other's welfare. It would be interesting to assess the degree of connection that an advantaged group feels to a disadvantaged group because greater feelings of connection with the outgroup might be associated with greater reports of group-based guilt. In the case of communal interpersonal relationships, the fear of rejection because of an interpersonal transgression leads to guilt. Therefore if an advantaged group feels connected to a disadvantaged group, then assessments of the anxiety or fear associated with rejection might also predict feelings of group-based guilt.

The present research represents an important advance in our understanding of the antecedents of group-based guilt. We chose to use specific examples of group differences that uniquely applied to each group so that we could test the effects of evaluations of our antecedents within individuals, using multilevel modeling. By using a variety of differences, we took advantage of naturalistic, existing group differences rather than holding group differences constant or experimentally manipulating the extent to which group differences were brought to mind. We covered a wide variety of categories including group differences that occur in financial, historical, social and political realms, as well as in gender roles, treatment by authorities, freedom and help from others. ${ }^{3}$ Yet despite the diversity of specific group differences that we addressed, we cannot know whether the groups would differentially perceive the antecedents or whether the antecedents would have different associations with guilt if they were described more generally 
(e.g. Whites have many privileges that Blacks do not) or if participants were allowed to generate their own list of group differences. Future research could examine this possibility, and also build from our existing work by manipulating levels of inequality, responsibility and justifiability that appear in overall group differences to be either high or low. Doing so would further clarify whether there are mainly additive effects or whether being presented with high or low levels of the antecedents produces interactions.

In conclusion, feelings of group-based guilt can motivate prosocial behavior in many forms. As a result of feeling group-based guilt, Whites support affirmative action for Blacks (Swim \& Miller, 1999), and both Whites and heterosexuals take collective action on behalf of Blacks and non-heterosexuals (Mallett, et al., 2006). Yet feelings of group-based guilt are relatively rare compared to other group-based emotions (Branscombe et al., 2004). Given the beneficial consequences of group-based guilt for intergroup relations, it is important to understand what factors lead to feelings of group-based guilt. Our research reveals that perceived inequality, responsibility and justifiability consistently predict feelings of group-based guilt for Whites, men and women. Understanding the antecedents of group-based guilt across a variety of social groups brings us one step closer to improving intergroup relations.

\section{Notes}

1. In addition to testing the three contrasts, we also tested whether each group differed from the other. That is, we compared Whites to men, Whites to women and men to women. Again, none of the contrasts significantly predicted the intercept of any antecedent or of group-based guilt.

2. To test whether gender significantly affected the interaction in the White data set, we added gender of participant as a level 2 predictor. It was not significant $(G=.03, S E=.11, p=\mathrm{ns})$.

3. To test whether our results varied by the type of group difference, we conducted separate analyses on each subgroup of differences. For example, for Whites, we compared financial differences to historical, social and political differences. Our results replicated across all subgroups for Whites and women, and across three of the five subgroups for men. This suggests that although we did not present Whites, women and men with identical group differences, the pattern of results that we present replicates across the same categories of group differences for many social groups.

\section{Acknowledgments}

The authors wish to thank Roger Giner-Sorolla and two anonymous reviewers for their comments on previous versions of this manuscript.

\section{References}

Anderson, N. B. (1989). Racial differences in stress-induced cardiovascular reactivity and hypertension: Current status and substantive issues. Psychological Bulletin, 105, 89-105.

Austin, W., \& Walster, E. (1974). Participants' reactions to 'equity with the world'. Journal of Experimental Social Psychology, 10, 528-548.

Baumeister, R. F., Stillwell, A. M., \& Heatherton, T. F. (1994). Guilt: An interpersonal approach. Psychological Bulletin, 115, 243-267.

Branscombe, N. R. (1998). Thinking about one's gender group's privileges or disadvantages: Consequences for well-being in women and men. British Journal of Social Psychology, 37, 167-184.

Branscombe, N. R., Doosje, B., \& McGarty, C. (2002). Antecedents and consequences of collective guilt. In D. M. Mackie \& E. R. Smith (Eds.), From prejudice to intergroup emotions: Differentiated reactions to social groups (pp. 49-66). New York: Psychology Press.

Branscombe, N. R., \& Miron, A. M. (2004). Interpreting the ingroup's negative actions toward another group: Emotional reactions to appraised harm. In L.Z. Tiedens \& C. W. Leach (Eds.), The social life of emotions. New York: Cambridge University Press.

Branscombe, N. R., Slugoksi, B., \& Kappen, D. M. (2004). The measurement of collective guilt. In N.R. Branscombe \& B. Doosje (Eds.), Collective guilt: International perspectives. (pp. 16-34). Cambridge, UK: Cambridge University Press.

Doosje, B., Branscombe, N. R., Spears, R., \& Manstead, A. (1998). Guilty by association: When one's group has a negative history. Journal of Personality and Social Psychology, 75, 872-886. 
Doosje, B., Branscombe, N. R., Spears, R., \& Manstead, A. (2004). Consequences of national ingroup identification for responses to immoral historical events. In N. R. Branscombe \& B. Doosje (Eds.), Collective guilt: International perspectives (pp. 95-111). Cambridge, UK: Cambridge University Press.

Estrada-Hollenbeck, M., \& Heatherton, T. F. (1998). Avoiding and alleviating guilt through prosocial behavior. In J. Bybee (Ed.), Guilt and children (pp. 215-231) San Diego, CA: Academic Press.

Glick, P., \& Fiske, S. T. (1997). Hostile and benevolent sexism: Measuring ambivalent sexist attitudes toward women. Psychology of Women Quarterly, 21, 119-135.

Harder, D. W., \& Greenwald, S .R. (1999). Further validation of the shame and guilt scales of the harder personal feelings questionnaire. Psychological Reports, 85, 271-281.

Hoffman, M. L. (1994). The contribution of empathy to justice and moral judgment. In B. Puka (Ed.), Reaching out: Caring, altruism, and prosocial behavior: Vol. 7. Moral development: A compendium (pp. 161-194). New York: Garland.

Iyer, A., Leach, C. W., \& Pederson, A. (2004). Racial wrongs and restitutions: The role of guilt and other group-based emotions. In

N. R. Branscombe \& B. Doosje (Eds.), Collective guilt: International perspectives (pp. 262-283). Cambridge, UK: Cambridge University Press.

Lerner, M. J. (1981). The justice motive in human relations: Some thoughts on what we know and need to know about justice. In M. J. Lerner \& S. C. Lerner (Eds.), The justice motive in social behavior. New York: Plenum.

Mallett, R. K., Huntsinger, J. R., Sinclair, S., \& Swim, J. K. (2006). Seeing through their eyes: When majority group members take collective action on behalf of an outgroup. Manuscript submitted for publication.

McIntosh, P. (1998). White privilege: Unpacking the invisible knapsack. In M. McGoldrick (Ed.), Re-visioning family therapy: Race, culture, and gender in clinical practice (pp. 147-152). New York: Guilford.

Miron, A. M., Branscombe, N. R., \& Schmitt, M. T. (2006). Collective guilt as distress over illegitimate intergroup inequality. Group Processes E Intergroup Relations, 9, 163-180.

Montada, L., \& Schneider, A. (1989). Justice and emotional reactions to the disadvantaged. Social Justice Research, 3, 313-344.
Monteith, M. J., Devine, P. G., \& Zuwerink, J. R. (1993). Self-directed versus other-directed affect as a consequence of prejudice-related discrepancies. Journal of Personality and Social Psychology, 64, 198-210.

Raudenbush, S. W., Bryk, A. S., Cheong, Y. F., \& Congdon, R. (2004). HLM 6: Hierarchical linear and nonlinear modeling. Chicago: Scientific Software International.

Rensmann, L. (2004). Collective guilt, national identity, and political processes in contemporary Germany. In N. R. Branscombe \& B. Doosje (Eds.), Collective guilt: International perspectives (pp. 169-190). Cambridge, UK: Cambridge University Press.

Roseman, I. J. (1984). Cognitive determinants of emotions. A structural theory. In P. Shaver (Ed.), Review of personality and social personality, 5, 11-35.

Russo, N. F. (1995). Women's mental health: Research agenda for the twenty-first century. In C. V. Willie, P. P. Rieker, B. M. Kramer, \& B. S. Brown (Eds.), Mental health, racism, and sexism. Pittsburgh, PA: University of Pittsburgh Press.

Schmidt, M. T., Branscombe, N. R., \& Brehm, J. W. (2004). Gender inequality and the intensity of men's collective guilt. In N. R. Branscombe \& B. Doosje (Eds.), Collective guilt: International perspectives (pp. 75-92). Cambridge, UK: Cambridge University Press.

Sprecher, S. (1986). The relation between inequity and emotions in close relationships. Social Psychology Quarterly, 49, 309-321.

Swim, J. K., Aikin, K. J., Hall, W. S., \& Hunter, B. A. (1995). Sexism and racism: Old-fashioned and modern prejudices. Journal of Personality and Social Psychology, 68, 199-214.

Swim, J. K., \& Miller, D. L. (1999). White guilt: Its antecedents and consequences for attitudes toward affirmative action. Personality and Social Psychology Bulletin, 25, 500-514.

Turner, C. B., \& Kramer, B. M. (1995). Connections between racism and mental health. C. V. Willie, P. P. Rieker, B. M. Kramer, \& B. S. Brown (Eds.), Mental health, racism, and sexism. Pittsburgh, PA: University of Pittsburgh Press.

Tyler, T. R., Boeckmann, R. J., Smith, H. J., \& Huo, Y. J. (1997). Social justice in a diverse society. Boulder, CO: Westview Press.

Walster, E., \& Walster, G. W. (1975). Equity and social justice. Journal of Social Issues, 31, 21-43.

Walster, E., Walster, G. W., Berscheid, E., Austin, W. Traupmann, J., \& Utne, M. K. (1978). Equity: Theory and Research. Boston: Allyn and Bacon. 
Paper received 5 December 2005; revised version accepted 3 August 2006.

\section{Biographical notes}

ROBYN K. MALLETT is a research assistant professor of psychology at the University of Virginia. Her research examines ways to optimize intergroup relations, ways to minimize the consequences of discrimination, and how individual differences in perceptions of prejudice and identification influence intergroup attitudes and ideologies.

JANET K. SWIM is a professor of psychology at the Pennsylvania StateUniversity. Her research includes judgments of prejudice and discrimination, affective and behavioral reactions to everyday forms of prejudice and discrimination, and parental socialization regarding race/ethnicity. 